\title{
Structural, spatial, and age effects on visual threshold
}

\author{
RICHARD WAXMAN \\ Touro College, New York, New York 10016 \\ and \\ ERIC KARP \\ York College, Jamaica, New York 11432
}

\begin{abstract}
The aim of the current research was to determine whether observers who are simultaneously presented with structural and spatial cues would demonstrate differential sensitivity to the structural characteristics of the stimulus, the spatial relationships between the stimulus and the visual field, or some interaction between these variables. A concurrent aim of the experiment was to determine the effects of aging on visual form detection. The results indicate that in the case of structural characteristics alone, subjects of all ages were influenced by size rather than visual angle or sidedness. The examination of the independent effect of spatial characteristics upon visual thresholds of younger and older subjects demonstrated greater observer sensitivity to parafoveal positions on the horizontal plane above the central horizontal meridian independent of which half visual field was stimulated. When the simultaneous effects of structural and spatial characteristics on sensitivity of younger and older subjects were observed, it was determined that position rather than size influences visual form detection. Although significant age differences were obtained between the two groups, both age groups were similarly influenced by the independent and interactive effects of structural and spatial characteristics.
\end{abstract}

Previous investigators have identified structural and spatial characteristics of stimuli that affect visual form detection. Bitterman, Krauskopf, and Hochberg (1954) demonstrated that the size of a visually presented geometric stimulus affects sensitivity for visual form detection. Michels and Zusne (1965) and Zusne (1970) reported that perception of complex forms depends on the number of sides contained in the stimulus. Other structural characteristics, such as symmetry, figural compactness, and the degree of variability in the size of the stimulus angles, have been shown to affect detection of complex forms. Howard and Templeman (1966) reported that spatial orientation of visual patterns affects stimulus detection. It was demonstrated that pairs of visual targets presented for discrimination in the vertical plane are more easily discriminated than the same pairs of stimuli presented in the horizontal plane.

The aim of the current research was to determine whether sensitivity for visual form detection would be more influenced by either structural or spatial characteristics. Specifically, it was questioned whether simultaneous presentation of structural and spatial characteristics would demonstrate greater subject sensitivity to structural characteristics, spatial characteristics, or some combination of the two.

Three simple stimuli in the form of triangles, circles, and squares were chosen for detection. Structurally, the stimuli were equated for visual angle but differed in size and sidedness. It was questioned which, if any, structural characteristics would significantly affect detection. Spatial effects were achieved by randomly presenting stimuli in any one of four predesignated parafoveal positions.

In addition, we wished to determine whether the age of the subject would affect sensitivity. The question was raised whether healthy subjects who were matched for visual acuity but differed in terms of age would show differential sensitivity to visual stimuli.

\section{METHOD}

\section{Subjects}

The performance of 10 male subjects (mean age $=35$ years) ranging in age from 19 to 61 years were examined. The subjects were drawn from the undergraduate student population at Touro College, which is located in New York City, and from the outpatient Psychological Services Clinic at the Brooklyn Veterans Administration Medical Center. All subjects were healthy and exhibited normal or corrected-to-normal (i.e., 20/20) visual acuity as measured by the Snellen chart.

\section{Apparatus}

The apparatus used in the experiment was the Scientific Prototype Model N-900 two-channel tachistoscope. The viewing distance as $83.8 \mathrm{~cm}$. The vertical field was $9.5 \mathrm{~cm}$ and subtended a visual angle of 6 deg $20 \mathrm{~min}$. The horizontal field was $12.8 \mathrm{~cm}$ and subtended a visual angle of $8 \mathrm{deg} 40 \mathrm{~min}$. The fixation spot was $5 \mathrm{~mm}$ and subtended a visual angle of $20 \mathrm{~min}$.

The stimulus field was divided into quadrants. The horizontal dimension of each quadrant was $4.75 \mathrm{~cm}$ and subtended a visual 
angle of $3 \mathrm{deg} 90 \mathrm{~min}$. The diagonal of each quadrant was $8 \mathrm{~cm}$ and subtended a visual angle of $5 \mathrm{deg} 30 \mathrm{~min}$.

Sequencing was controlled by three timers ranging from $1 \mathrm{msec}$ to $9.9 \mathrm{sec}$. All sequencing and timing operations were silent.

Intensity was controlled by current-feedback regulators for $1-\log$-unit attenuation from a maximum of $30 \mathrm{fL}$ and was set at $50 \%$ maximum intensity, with the red fixation spot set at maximum intensity, for each presentation.

\section{Stimuli}

All stimuli were $1 \mathrm{~cm}$ in width and subtended a visual angle of $40 \mathrm{~min}$. Stimuli were circles, squares, and triangles traced as 1-mm black lines on a white background. Stimuli were presented on the 45 th meridian, between $3.5 \mathrm{~cm}(2 \mathrm{deg} 20 \mathrm{~min})$ and $4.5 \mathrm{~cm}(3 \mathrm{deg})$ from fixation in each quadrant. The area of the triangle equaled $50 \mathrm{~mm}^{2}$. The area of the circle equaled $88.5 \mathrm{~mm}^{2}$.

\section{Procedure}

Each subject was first instructed to look into the tachistoscope, and all possible stimulus choices were randomly presented in a monocular fashion for $3 \mathrm{sec}$. Following each presentation, the subject was instructed to either verbalize or point to the target perceived on a stimulus choice card. Once the subject adequately demonstrated the ability to identify stimuli, the experiment began.

Each subject was given monocular presentations of four stimulus possibilities (i.e., circle, square, triangle, or blank in each quadrant). A tone that signaled the upcoming presentation was presented to the subjects. The termination of the tone initiated a preadapting field and fixation spot for a duration of $2 \mathrm{sec}$. Termination of the preadapting field triggered the stimulus field. The duration of the target presentation depended on the threshold attainment.

Visual threshold in the current study was defined as the minimum tachistoscopic exposure necessary for detection $66 \%$ of the time the stimulus was actually presented.

\section{RESULTS}

The purpose of this research was to examine the independent and interdependent effects of structural and spatial characteristics on visual form detection, and also to determine if a subject's age plays any significant role in such detection.

An analysis of variance was performed to arrive at the results that are shown in Table 1. A first-order analysis of variance examining the effects of stimulus structure on visual threshold for young and old subjects proved significant $[F(2,32)=9.685, \mathrm{p}<.01]$. The differences among the cumulative mean scores for the triangle, circle, and square resulted in the obtained variance. The mean threshold for the triangle whose area equaled $50 \mathrm{~mm}^{2}$ was $10.42 \mathrm{msec}$. The mean threshold for the circle whose area equaled $88.5 \mathrm{~mm}^{2}$ was $9.32 \mathrm{msec}$. The mean threshold for the square whose area equaled $100 \mathrm{~mm}^{2}$ was $9.21 \mathrm{msec}$.

A first-order age-independent analysis of variance examining the effects of the quadrant position (i.e., horizontal fixed positions above or below the center horizontal meridian) proved to be significant $[F(1,16)=$ $24.023, \mathrm{p}<.01]$. The cumulative mean thresholds that,
Table 1

Analysis of Variance Examining the Effect of Age, Shape, Quadrant Position, and Visual Field Position on Visual Form Detection

\begin{tabular}{lrcrc}
\hline \multicolumn{1}{c}{ Source } & \multicolumn{1}{c}{ SS } & df & \multicolumn{1}{c}{ MS } & \multicolumn{1}{c}{ F } \\
\hline Stimulus (S) & 72.16 & 2,32 & 36.06 & $9.6850^{*}$ \\
Quadrant (Q) & 222.92 & 1,16 & 222.92 & $24.0230^{*}$ \\
Visual Field (V) & 4.19 & 1,16 & 4.19 & .0247 \\
Group (G) & 2963.15 & 1,16 & 2963.15 & $17.0270^{*}$ \\
Q by S & 102.67 & 2,32 & 51.33 & $52.4270^{*}$ \\
V by S & 23.45 & 2,32 & 11.73 & 3.1490 \\
G by S & 6.91 & 2,32 & 3.46 & .9280 \\
G by Q & 16.91 & 1,16 & 16.91 & 1.8230 \\
G by V & 7.11 & 1,16 & 7.11 & .0410 \\
\hline
\end{tabular}

${ }^{*} p<.01$.

when analyzed, resulted in the obtained significance were $8.69 \mathrm{msec}$ for the upper quadrants and $10.62 \mathrm{msec}$ for the lower quadrants.

When the effects of visual field location were examined (i.e., vertically fixed positions to the left or right of the center vertical meridian), no significant differences in the threshold were found $[\mathrm{F}(1,16)=.0247$, $\mathrm{p}<.05]$.

The age of the subjects significantly affected visual threshold $[F(1,16)=17.027, p<.01]$. The cumulative mean threshold score for the younger group equaled $6.14 \mathrm{msec}$. The cumulative mean threshold score for the older group equaled $13.6 \mathrm{msec}$.

A second-order analysis of variance examining the interaction between the quadrant and stimulus variables proved significant $[F(2,32)=52.427, p<.01]$. In order to ascertain the source of variance, the cumulative age combined mean thresholds for three geometric stimuli were analyzed according to their positions in the visual field. The results are shown in Table 2 .

While the findings show a significant interaction between quadrant and the stimulus variable (i.e., horizontal position), no significant interactive effects were obtained between visual field (vertical position and stimulus) and the stimulus variables $[\mathrm{F}(2,32)=3.149$, $\mathrm{p}<.05]$.

Second-order analyses of variance examining the possible role of aging and horizontal position, vertical position, or stimulus type upon form detection proved nonsignificant (the results are shown in Table 1).

Table 2

Cumulative Mean Thresholds for Three Geometric Stimuli as Analyzed According to Quadrant Position

\begin{tabular}{|c|c|c|c|c|}
\hline Quadrant & $\begin{array}{c}\text { Triangle } \\
\mathrm{A}=50 \mathrm{~mm}^{2}\end{array}$ & $\begin{array}{c}\text { Circle } \\
-88.5 \mathrm{~mm}^{2}\end{array}$ & $\begin{array}{c}\text { Square } \\
-100 \mathrm{~mm}^{2}\end{array}$ & Mean \\
\hline Upper & 10.36 & 7.71 & 7.99 & 8.69 \\
\hline Lower & 10.49 & 10.93 & 10.43 & 10.62 \\
\hline Mean & 10.42 & 9.32 & 9.21 & 9.65 \\
\hline
\end{tabular}




\section{DISCUSSION}

Previous research has demonstrated that visual form detection of complex shapes is affected by structural and spatial stimulus characteristics. The purpose of the current research was to determine whether observers who are simultaneously stimulated by structural and spatial characteristics of simple geometric figures would dèmonstrate greater sensitivity to structural characteristics of the stimulus, spatial relationships between the stimulus and the visual field, or some interaction between structural and spatial phenomena.

Prior to determining the presence or absence of any of these effects, it was necessary to determine to which structural and spatial characteristics (independent of each other) the subjects were responding.

The results demonstrate that subjects were sensitive to the size rather than to the visual angle or the number of sides of the stimulus. Generally, as stimulus size increased, threshold decreased. These data were comparable to results obtained by Bitterman et al. (1954). Earlier reports by Zusne (Michels \& Zusne, 1965; Zusne, 1970) that demonstrated the relationship between sidedness and visual threshold may reflect different perceptual strategies necessary for detection of complex, nonlabelable geometric stimuli.

Examination of spatial effects on visual threshold demonstrated that observers were more sensitive to stimuli presented in the upper rather than lower quadrants regardless of which half of the visual field was shown the stimuli. Healthy subjects with normal visual acuity may possess selective zones of spatial sensitivity in their visual fields. This phenomenon may be expressed as greater observer sensitivity for positions on the horizontal plane above the central horizontal meridian.

When presented visual stimuli, subjects appeared to be more responsive to the position of the stimulus in the visual field than to its size. The results demonstrate that while size generally affects threshold, this phenomenon dissipates when form detection is analyzed according to location of the stimulus in the visual field. Independent of stimulus size and in which half visual field stimuli were presented, stimuli were more detectable in the upper than in the lower quadrants. Stimulus size by itself does affect threshold. However, when its effects are examined in relation to stimulus position in the visual field, size appears to be more of a factor in the upper quadrants. In the lower quadrants, size does not appear to be a significant factor in relation to threshold.

A second major concern of the experiment was to determine age effects on identification. Older subjects required stimulus exposures that were about twice as long as the younger subjects required in order to identify the stimuli. Nonetheless, the older subjects responded in patterns similar to the younger group of subjects. That is, generally, size did affect threshold. Stimuli presented in the upper quadrants were more readily perceived than stimuli presented in the lower quadrants. The older subjects identified the stimuli equally well independent of which visual semifield was stimulated.

These results suggest that visual sensitivity in the older group of subjects is reflective of the slower processing time that is characteristic of an "older" nervous system. Older subjects may need longer presentations in order to extract information necessary for stimulus detection. Botwinick (1978) suggested that stimulation via any modality may linger in the central nervous system of older individuals. This persistance may correspond to the increased presentation time needed by older subjects to reach threshold.

\section{REFERENCES}

Bitterman, M. E., Krauskopf, J., \& Hochberg, J. E. Threshold for visual form: A diffuse model. American Journal of Psychology, 1954, 67, 205-219.

Botwinick, J. Aging and behavior. New York: Springer, 1978.

Howard, I., \& Templeton, W. B. Human spatial orientation. London: Wiley, 1966.

Michels, K., \& ZuSne, L. Metrics of visual form. Psychological Bulletin, 1965, 63, 74-86.

ZuSNe, L. Visual perception of form. New York: Academic Press, 1970.

(Received for publication August 10, 1981.) 